\title{
A six-week motor-driven functional electrical stimulation rowing program improves muscle strength and body composition in people with spinal cord injury: a pilot study
}

D-I Kim, D-S Park, BS Lee and JY Jeon

Spinal Cord (2014) 52, 785; doi:10.1038/sc.2014.133

Correction to: Spinal Cord (2014) 52, 621-624; doi:10.1038/ sc.2014.76; published online 3 June 2014

Since the publication of this paper, the authors have noticed that FES was incorrectly expanded as functional electronic stimulation instead of functional electrical stimulation.

The authors apologize for any inconvenience caused. 